\title{
On the Design of Rate Adaptation for Relay-Assisted Satellite Hybrid FSO/RF Systems
}

This paper was downloaded from TechRxiv (https://www.techrxiv.org).

\section{LICENSE}

CC BY 4.0

SUBMISSION DATE / POSTED DATE

$17-08-2021 / 20-08-2021$

\section{CITATION}

Le, Hoang; Nguyen, Thang (2021): On the Design of Rate Adaptation for Relay-Assisted Satellite Hybrid FSO/RF Systems. TechRxiv. Preprint. https://doi.org/10.36227/techrxiv.15177573.v1

DOI 


\title{
On the Design of Rate Adaptation for Relay-Assisted Satellite Hybrid FSO/RF Systems
}

\author{
Thang V. Nguyen, Student Member, IEEE, Hoang D. Le, Student Member, IEEE, Ngoc T. Dang, Member, IEEE \\ and Anh T. Pham, Senior Member, IEEE
}

\begin{abstract}
This paper addresses the design of hybrid freespace optical/radio frequency (FSO/RF) systems for a highaltitude platform (HAP)-aided relaying satellite communication for mobile networks supported by unmanned aerial vehicle (UAV). While prior work primarily focused on fixed-rate design, which frequently switches between FSO and RF lead to reduce the system performance, we propose a rate adaptation design that gradually adjusts the data rate in each link when its channel state fluctuates. The proposed design's downlink performance is analyzed, taking into account many challenging issues, including beam spreading loss, cloud attenuation, statistical behaviors of the atmospheric turbulence in the dual-hop channel, and pointing misalignment due to the UAV hovering. Different performance metrics are analytically derived based on channel modelings, such as outage probability, average transmission rate, achievable spectrum efficiency, and average transmission rate. The numerical results quantitatively confirm the effectiveness of our proposed system under the impact of UAV hovering misalignment and atmospheric-related issues like clouds and turbulence. Finally, Monte-Carlo simulations validate the accuracy of theoretical results.
\end{abstract}

Index Terms-Satellite communications, High-altitude platform (HAP), unmanned aerial vehicles (UAVs), Adaptive rate, Hybrid FSO/RF systems.

\section{INTRODUCTION}

According to the International Telecommunication Union (ITU) report in 2019, half of the world population did not have the regular Internet access [1]. The main barriers come from a lack of infrastructure and affordability. Satellite communications have recently emerged as a complementary solution to bridge the gap by offering Internet connectivity at a reasonable cost and ubiquitous coverage [2]. For example, Starlink presently offers Internet access from space with a fee of 99 USD per month (unlimited data); download speed varies from 50 to $150 \mathrm{Mbps}$ depending on the location [3].

As satellite communication is susceptible to weather-related issues (e.g., clouds, rain, atmospheric turbulence), it is still a challenge to increase the data rate and the coverage area. A promising solution is to use a high-altitude platform (HAP), which is a quasi-stationary vehicle located at a cloud-free atmospheric altitude from 17 to $25 \mathrm{~km}$ above the earth's surface [4]. Thanks to unique properties, such as quick deployment, low maintenance cost, and broader coverage, the

Thang V. Nguyen, Hoang D. Le, and Anh T. Pham are with the Computer Communications Laboratory, the University of Aizu, Aizuwakamatsu 965-8580, Japan (email: nguyenvanthang2404@gmail.com, hoangbkset@gmail.com,pham@u-aizu.ac.jp).

Ngoc T. Dang is with the Posts and Telecommunications Institute of Technology, Hanoi 100000, Vietnam (email: ngocdt@ptit.edu.vn).

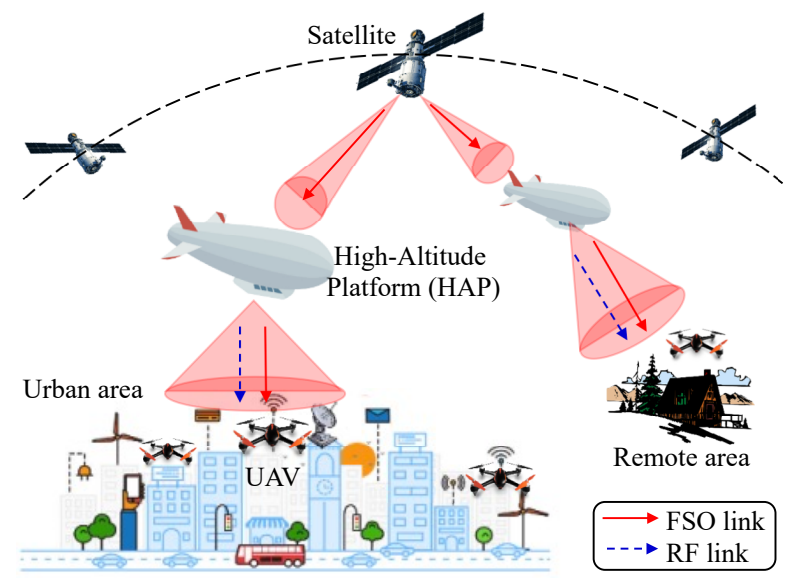

Fig. 1. HAP-assisted satellite network with UAVs.

development of HAP-assisted satellite can further improve the scalability of $5 \mathrm{G}$ network and beyond [5]-[7]. In HAPassisted satellite network, a swarm of HAP can be deployed to cover a vast area, e.g., approximately $100 \mathrm{~km}$ as expected by Softbank [8]. Besides, HAP can be a stand-alone system to provide a stable connection to a remote area, temporary link to emergencies situations, such as a natural disaster, as described in Fig. 1. Furthermore, the use of unmanned aerial vehicles (UAVs) as mobile base stations can further enhance the Internet connectivity provision as they can flexibly adjust their positions based on the demand on the ground. The UAVmounted mobile base station solution has gained significant interest in both academic and industry [9]-[12]. In case of natural disaster, UAVs can promptly give warnings, rapidly assist rescue and temporary recover the operation of damaged communication networks [9].

In the considered network, HAP functions as a relay station between the satellite and UAVs. The system, therefore, consists of two links: (1) a satellite-to-HAP and (2) HAP-to-UAV links. As the HAP situates in the stratosphere, which is less susceptible to atmospheric turbulence and clouds, we employ a free-space optical (FSO) link from satellite to HAP for a highspeed connection. In the second hop between HAP and UAVs, the FSO link may face weather-related issues such as clouds, snow, haze, rain, atmospheric turbulence, etc., which reduce link availability and limit the link distance [13]. In addition, multiple UAVs can be supported by one HAP; UAVs' locations are therefore not necessarily in the center of the optical beam. Also, UAVs can be in mobility; the optical link outage may 
occur because of the combined effect of beam misalignment and UAV hovering. Therefore, a hybrid RF/FSO system is proposed, in which the FSO link is prioritized higher. The RF is used as a backup link when the optical channel condition is insufficient to maintain the high data rate.

As a matter of fact, the dual-hop relay-assisted FSO and hybrid FSO/RF communications have been well studied for terrestrial networks [14]-[17]. In the satellite networks, the current state-of-the-art of hybrid FSO/RF systems for relayassisted satellite communication focuses on fixed users [18] [22]. In particular, in [18], the authors studied the downlink satellite-HAP-ground with the assumption of gamma-gamma turbulence channel model for FSO link and Rician distribution for RF link. The impact of pointing error and atmospheric attenuation was not included in the study. The hybrid FSO/RF for uplink ground-HAP-satellite scenarios was considered in [19], in which the RF channel was also modeled by Rician distribution. The combined channel model for the FSO link was taken free space loss, atmospheric turbulence, and beam wander induced pointing error into account. In both [18], [19], the single-threshold-based scheme was used to switch between RF and FSO links. The adaptive-combining-based switching scheme was used for integrated satellite-HAP-ground with hybrid FSO/RF system in both cases of uplink and downlink [20], [21]. The maximal-ratio-combining of FSO and RF links was implemented at the receiver when the RF link was transmitted simultaneously with the FSO link. Nevertheless, all previous work did not consider the case of mobile users, such as UAV-mounted mobile base stations, which are being considered for irregular, unexpected events, including sports events, natural disasters, and emergencies. In addition, the adaptive rate hybrid FSO/RF system was recently applied for the downlink satellite-HAP-ground [22]. With the help of the rate adaptation, the hybrid FSO/RF system can avoid frequently switching between FSO link and RF link, leading to enhancing the system performance.

In this paper, we, therefore, attempt to provide a comprehensive design and an insightful evaluation of dual-hop satelliteHAP-UAV hybrid FSO/RF systems with rate adaptation for mobile users, which can be summarized as follows:

- Firstly, we propose a multi-rate design for the integrated downlink satellite-HAP-UAV with the help of hybrid FSO/RF systems to counteract the effect of transmission media. As the role of the relay node, the amplified and forward scheme is implemented at HAP thanks to its cost effectiveness and simple hardware requirements.

- Secondly, a number of performance metrics, including the end-to-end outage probability, average transmission rate, achievable spectrum efficiency, and average bit error rate, have been derived considering various atmosphericrelated issues. Specifically, the Rician distribution is used to model the RF channel. The FSO channel is modeled by taking into account the atmospheric turbulence, which is modeled by Gamma-Gamma distribution, cloud attenuation, beam spreading loss, and UAV hovering misalignment. The Monte-Carlo simulation verifies the accuracy of the theoretical model.

- Finally, from the perspective of practical design, the insightful numerical results are discussed to quantify the effect of transmission media on the dual-hop satelliteHAP-UAV hybrid FSO/RF system. To this end, we have demonstrated the effectiveness of the proposed system compared with the conventional ones.

The rest of the paper is organized as followed. The relayassisted satellite hybrid FSO/RF communication system description is shown in Section II. In Section III, the FSO and RF channel models are investigated. Next, Section IV presents rate adaptation and analyzes the system performance concerning the average transmission rate, achievable spectrum efficiency, outage probability, and average bit error rate. The discussion on the numerical results is expressed in Section V. Finally, the critical points of our work are concluded in Section VI.

\section{SYSTEM DESCRIPTIONS}

Figure 2 describes a block diagram of dual-hop satelliteHAP-UAV with an FSO system at first hop and hybrid FSO/RF system at the second hop. In the considered system, the satellite is a source node. The HAP is a relay node that amplifies the received signal from the satellite then forwards it to the destination. The UAV-mounted base station is a destination node. At the transmitter, the data is modulated by a quadrature amplitude modulation (QAM) modulator, and the output signal can be expressed as

$$
s_{n}(t)=A_{n I} g(t) \cos \left(2 \pi f_{r} t\right)-A_{n Q} g(t) \sin \left(2 \pi f_{r} t\right),
$$

where $g(t)$ is the pulse shaping function, $f_{r}$ is the subcarrier frequency, $0 \leq t \leq T$ with $T$ is the interval time of the QAM symbol, $A_{n I}=\{2 n-1-I\}_{n=1}^{I}$ and $A_{n Q}=$ $\{2 n-1-Q\}_{n=1}^{Q}$ are the amplitude of the information signal belong to in-phase and quadrature, respectively. Then, the electrical QAM signal is used to modulate the intensity of optical beam to generate the SC-QAM optical signal, which can be shown as

$$
s(t)=P_{\mathrm{t}}\left[1+m s_{n}(t)\right],
$$

where $P_{\mathrm{t}}$ is the transmitted power at the satellite, $m$ is the modulation index. At the HAP, i.e., relay node, the optical aperture collects the optical signals then forwards them to an optical switch. Based on channel state information (CSI), the signal is decided to transmit RF or FSO link in the next state. If the RF link is selected, the optical signal is converted to the electrical signal by photodiode then amplify before transmitting to the destination node. If the FSO link is chosen, the optical signal is amplified and directly forward to the ground station. The representative of the output signal of the relay node can be expressed as

\section{For RF link:}

$$
r_{r}(t)=G_{\mathrm{RF}}\left[\eta h_{\mathrm{SR}} P_{\mathrm{t}} m s_{n}(t)+\eta n_{\mathrm{b} 1}+n_{\mathrm{re} \_ \text {hap }}\right],
$$

For FSO link:

$$
r_{f}(t)=G_{\mathrm{F}}\left[h_{\mathrm{SR}} s(t)+n_{\mathrm{b} 1}\right],
$$

where $\eta$ is the optical-to-electrical coefficient, $h_{\mathrm{SR}}$ is the beam spreading loss at the first hop, $n_{\mathrm{b} 1}$ is the background noise, 


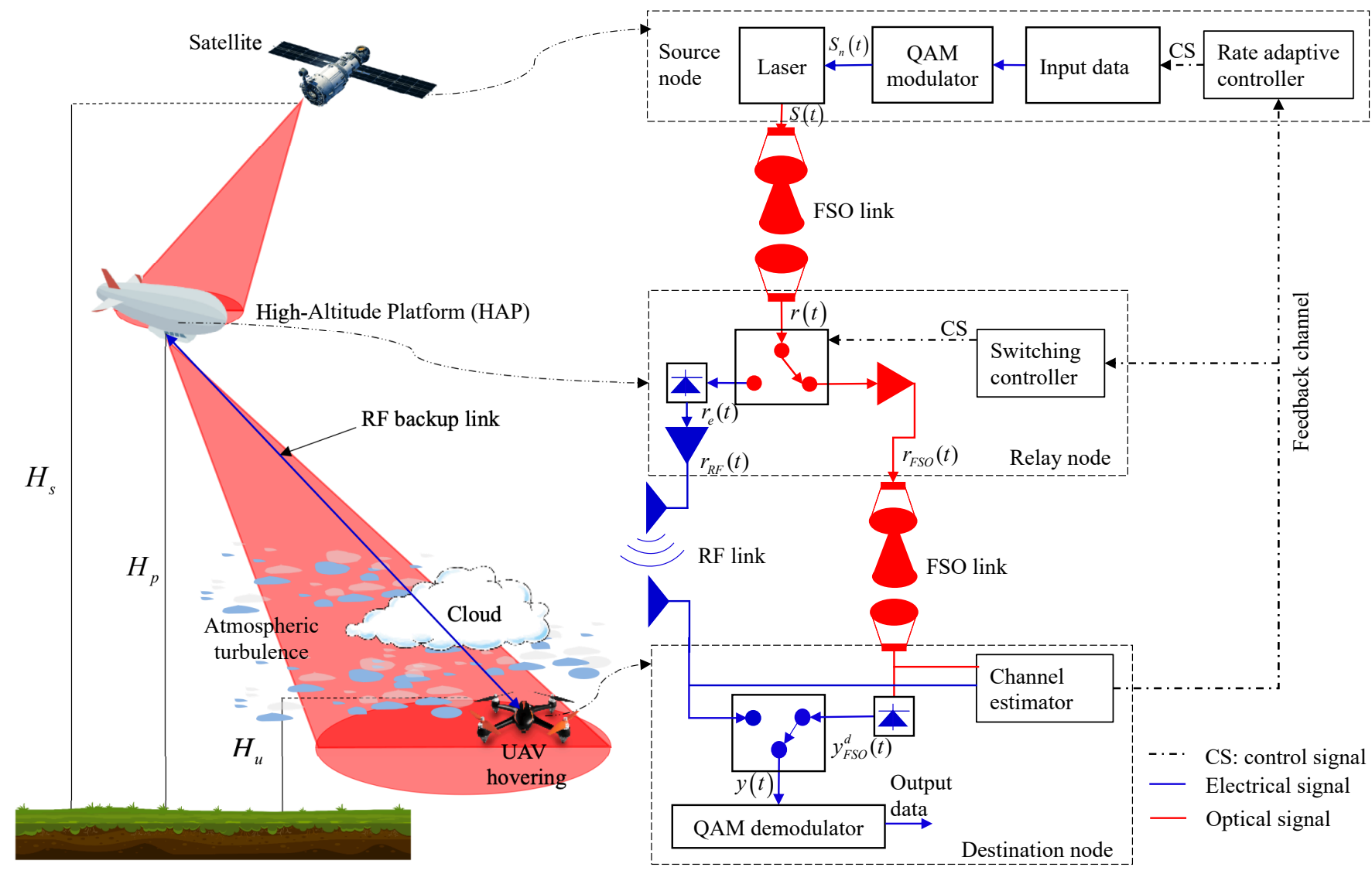

Fig. 2. The dual-hop satellite-HAP-UAV hybrid FSO-FSO/RF systems including challenge issues (left side) and block diagram (right side).

and $n_{\text {re_hap }}$ is the receiver noise, $G_{\mathrm{RF}}$ is the electrical amplifier gain, $G_{\mathrm{F}}$ is the optical amplifier gain. Based on CSI, the FSO link will be activated if the channel quality is good. Otherwise, the RF link will be applied if the channel condition is poor. The received signal at the ground station can be expressed as

For RF link:

$$
\begin{aligned}
y_{r}(t) & =h_{\mathrm{RF}} G_{\mathrm{RF}} \eta h_{\mathrm{SR}} P_{\mathrm{t}} m s_{n}(t)+h_{\mathrm{RF}} G_{\mathrm{RF}} \eta n_{\mathrm{b} 1} \\
& +h_{\mathrm{RF}} G_{\mathrm{RF}} n_{\text {re_hap }}+n_{\mathrm{re}} .
\end{aligned}
$$

\section{For FSO link:}

$$
\begin{aligned}
y_{f}(t) & =h_{\mathrm{FSO}} \eta G_{\mathrm{F}} h_{\mathrm{SR}} P_{\mathrm{t}} m s_{n}(t) \\
& +\eta h_{\mathrm{FSO}} G_{\mathrm{F}} n_{\mathrm{b} 1}+\eta n_{\mathrm{b} 2}+n_{\mathrm{rec}}
\end{aligned}
$$

where $h_{\mathrm{RF}}$ and $h_{\mathrm{FSO}}$ are the channel coefficient of the RF link and the FSO link, respectively. $n_{\text {re }}$ and $n_{\text {rec }}$ are receiver noise. For convenient, $G_{\mathrm{RF}}^{2}=\frac{1}{\left(P_{\mathrm{t}} m h_{\mathrm{SR}}\right)^{2}+\left(\sigma_{\mathrm{b} 1}\right)^{2}}$ and $G_{\mathrm{F}}^{2}=$ $\frac{1}{\left(P_{\mathrm{t}} m h_{\mathrm{SR}}\right)^{2}+\left(\sigma_{\mathrm{b} 1}\right)^{2}}$, the SNR at the destination node, denoted as $\gamma_{f}^{e 2 e}$ for FSO-FSO link and $\gamma_{r}^{e 2 e}$ for FSO-RF link, can be given as

For FSO-RF link:

$$
\begin{aligned}
\gamma_{e 2 e}^{r} & =\frac{\left(h_{\mathrm{RF}} G_{\mathrm{RF}} \eta h_{\mathrm{SR}} P_{\mathrm{t}} m\right)^{2}}{\left(h_{\mathrm{RF}} G_{\mathrm{RF}} \eta \sigma_{\mathrm{b} 1}\right)^{2}+\left(h_{\mathrm{RF}} G_{\mathrm{RF}} \sigma_{\text {re_hap }}\right)^{2}+\left(\sigma_{\mathrm{re}}\right)^{2}}, \\
& =\frac{\gamma_{1} \gamma_{2, \mathrm{RF}}}{\gamma_{1}+\gamma_{2, \mathrm{RF}}+1} .
\end{aligned}
$$

\section{For FSO-FSO link:}

$$
\begin{aligned}
\gamma_{e 2 e}^{f} & =\frac{\left(h_{\mathrm{FSO}} \eta G_{\mathrm{F}} h_{\mathrm{SR}} P_{\mathrm{t}} m\right)^{2}}{\left(h_{\mathrm{FSO}} \eta G_{\mathrm{F}} \sigma_{\mathrm{b} 1}\right)^{2}+\left(\eta \sigma_{\mathrm{b} 2}\right)^{2}+\left(\sigma_{\mathrm{rec}}\right)^{2}}, \\
& =\frac{\gamma_{1} \gamma_{2, \mathrm{FSO}}}{\gamma_{1}+\gamma_{2, \mathrm{FSO}}+1} .
\end{aligned}
$$

where $\gamma_{1}=\frac{\left(P_{\mathrm{t}} m h_{\mathrm{SR}}\right)^{2}}{\left(\sigma_{\mathrm{b} 1}\right)^{2}}$ is the SNR of FSO link at the first hop. In the second hop, $\gamma_{2, \mathrm{RF}}=\frac{\left(\eta h_{\mathrm{RF}}\right)^{2}}{\left(h_{\mathrm{RF}} G_{\mathrm{RF}} \sigma_{\mathrm{re}-}-{ }_{\mathrm{hap}}\right)^{2}+\left(\sigma_{\mathrm{re}}\right)^{2}}$ is the SNR of the RF link. And, $\gamma_{2, \mathrm{FSO}}=\frac{\left(\eta h_{\mathrm{FSO}}\right)^{2}}{\left(\eta \sigma_{\mathrm{b} 2}\right)^{2}+\left(\sigma_{\mathrm{rec}}\right)^{2}}$ is the SNR of FSO link at the second hop.

\section{Channel Modeling}

The integrated satellite-HAP-UAV system considered in this paper contains two hops: (1) satellite-to-HAP using FSO links, and (2) HAP-to-UAV using hybrid FSO/RF links. This section investigates the challenging factors, such as clouds, atmospheric turbulence, pointing misalignment, UAV hovering, beam divergence loss, for each hop separately.

\section{A. Satellite-to-HAP FSO link}

Due to the relative motion between satellite and HAP, unfortunately, the Doppler effect may affect received signals. Nevertheless, the existing receiver design for optical satellite communication is able to deal with the Doppler frequency shift as reported in [23], [24]. Hence, the effect of Doppler 
can be negligible in our work. On the other hand, the impact of clouds and atmospheric turbulence on laser beam can be ignore since HAP situates at cloud-free altitude (i.e., 17-25 $\mathrm{km}$ ) as described above. Besides, an optical Gaussian beam is diverged by some amount over the path from the satellite to HAP. The divergence loss is computed as the ratio between the fraction of collected power of HAP's aperture and the area of beam footprint. For a single beam, the divergence loss can be approximately calculated as

$$
L_{g e o} \approx A_{0} \exp \left(-\frac{2 \rho^{2}}{w_{d e q}^{2}}\right),
$$

where $w_{d e q}^{2}=w_{d}^{2} \frac{\sqrt{\pi} \operatorname{erf}(v)}{2 v \exp \left(-v^{2}\right)}$ is the equivalent beam waist, in which $w_{d}=w_{0} \sqrt{1+\varepsilon\left(\frac{\lambda d_{1}}{\pi \omega_{0}^{2}}\right)^{2}}$ is the beam waist at the distance $d_{1}$, which is given by $d_{1}=\left(H_{s}-H_{p}\right) / \cos \left(\xi_{s}\right)$ in which $H_{s}$ is the altitude of the satellite, $H_{p}$ is the height of the HAP, and $\xi_{s}$ is the zenith angle of the satellite, $w_{0}=(2 \lambda) /\left(\pi \theta_{s}\right)$ is the beam waist at $d_{1}=0$ with $\theta_{s}$ is the divergence angle of the satellite, $\varepsilon=\left(1+2 \frac{w_{0}^{2}}{\rho_{0}^{2}\left(d_{1}\right)}\right)$, and $\rho_{0}\left(d_{1}\right)=\left(0.55 C_{n}^{2} k_{w}^{2} d_{1}\right)^{-3 / 5}$ is the coherence length [25]. In this study, we assume that active tracking between the satellite and the HAP is perfect, then the detector of HAP is located at the center of beam footprint (i.e., $\rho=0$ ). Hence, $A_{0}=[\operatorname{erf}(\mathrm{v})]^{2}$ corresponds to the fraction of the collected power at $\rho=0$ with $v=\frac{a \sqrt{\pi}}{w_{d} \sqrt{2}}$ is the ratio between aperture radius and beam-width, in which $a$ is the radius of detector.

\section{B. HAP-to-UAV hybrid FSO/RF link}

1) RF channel: The Rician distribution is used to model the RF channel due to line-of-sight (LOS) transmission between HAP and UAV. The channel coefficient of the RF link can be given as $h_{R F}=g_{1} h_{r}$, where $g_{1}$ and $h_{r}$ denote the average power gain and fading gain of the RF link, respectively. The power gain of the RF link can be expressed as

$$
g_{1}(d B)=G_{T}+G_{R}-L_{F}-L_{A}-L_{c},
$$

where $G_{T}$ and $G_{R}$ are the transmit and receive antenna gains, respectively. $L_{A}$ denotes the gaseous atmosphere loss [19]. $L_{F}=20 \log _{10}\left(\frac{4 \pi d_{2}}{f_{r}}\right)$ is the free space loss from the HAP to UAV, in which $f_{r}$ is the frequency for RF link, and $d_{2}=$ $\left(H_{p}-H_{u}\right) / \cos \left(\xi_{p}\right)$ is the transmission distance from HAP to UAV with HAP's zenith angle of $\xi_{p}$, and $H_{u}$ is the altitude of UAVs.

Cloud effects: As introduced by ITU-P840 [26], the cloud attenuation model is based on Rayleigh scattering suitable for frequencies up to $200 \mathrm{GHz}$, and it is expressed as

$$
L_{c}=d_{c} \alpha_{1}
$$

where $L_{c}$ is the attenuation due to effect of cloud, in which $d_{c}=d_{z} / \cos \xi_{p}$ is the signal path propagation in clouds, in which $d_{z}$ is the considered length of clouds. $\alpha_{1}=$ $K_{c} M_{c}$ is the specific attenuation within clouds in which $K_{c}$ $\left((\mathrm{dB} / \mathrm{km}) /\left(\mathrm{g} / \mathrm{m}^{3}\right)\right)$ is the specific attenuation coefficient, $M_{c}$ $\left(\mathrm{g} / \mathrm{m}^{3}\right)$ is the cloud liquid water content [26].
Rician fading model: Due to LOS between HAP and UAV, and following [27], [28], the fading gain is then modeled by Rician distribution with the probability density function (PDF) and cumulative density function (CDF) of the SNR received at the destination can be given respectively by

$$
\begin{aligned}
f_{\gamma_{2, R F}}\left(\gamma_{2, R F}\right) & =\frac{K+1}{\bar{\gamma}_{R F}} I_{0}\left(2 \sqrt{\frac{K(K+1) \gamma_{2, R F}}{\bar{\gamma}_{R F}}}\right) \\
& \times \exp \left[-\frac{(K+1) \gamma_{2, R F}}{\bar{\gamma}_{2, R F}}-K\right], \\
F_{\gamma_{2, R F}} & =1-Q_{1}\left(\sqrt{2 K}, \sqrt{\frac{2(K+1)}{\bar{\gamma}_{2, R F}} \gamma_{2, R F}}\right),
\end{aligned}
$$

where $Q_{1}(\cdot, \cdot)$ is the Marcum $Q_{1}$-function, $K$ is the Rician factor which reflects the relative strength of the direct LOS path component of fading coefficient. $\bar{\gamma}_{R F}$ is the average SNR of the RF link.

2) FSO channel: For the downlink from HAP to UAV, the mathematical model of channel coefficient is expressed as $h_{F S O}=h_{c} h_{a} h_{p}$ in which $h_{c}$ is the cloud attenuation, $h_{a}$ is turbulence-induced fading, and $h_{p}$ is UAV hovering-induced fading.

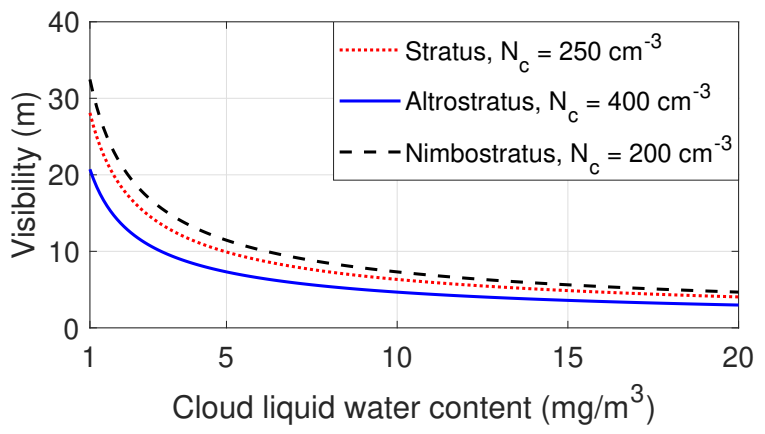

Fig. 3. An example of the visibility for several cloud types.

Cloud effects: In the optical downlink, atmospheric attenuation is severe due to scattering, which is caused by clouds [29]-[32]. Clouds result from the condensation of water vapors above the earth' s surface and may occasionally disrupt the signal or completely block the optical signal from HAP to UAV. Besides, optical wavelength, which operates in $\mathrm{THz}$ band corresponds to the range of wavelength from 0.8 to 2 $\mu m$, is about the same particle size of water vapors. Hence, the scattering phenomenon occurs on the beam propagation, leading to decrease visibility significantly and signal power [31], [32]. In this model, the visibility can be determined based on cloud droplet number concentration $N_{c}$ and cloud liquid water content $M_{c}$ as followed [33]

$$
V=\frac{1.002}{\left(N_{c} \times M_{c}\right)^{0.6473}} .
$$

The cloud effects on the visibility is illustrated in Fig. 3 for various cloud types such as stratus $\left(N_{c}=250 \mathrm{~cm}^{-3}\right)$, altrostratus $\left(N_{c}=400 \mathrm{~cm}^{-3}\right)$, and nimbostratus $\left(N_{c}=200 \mathrm{~cm}^{-3}\right)$. The figure reveals that visibility decreases rapidly when the 
CLWC grows up. Following the Beer-Lambert law, the cloud attenuation can be expressed [31]

$$
h_{c}=\exp \left(-\alpha_{2} d_{c}\right),
$$

where $d_{c}$ is the distance regarding scattering phenomena happen. $\alpha_{l}$ is the attenuation coefficient and can be expressed as

$$
\alpha_{2}=\frac{3.91}{V[k m]}\left(\frac{\lambda[n m]}{550}\right)^{-q},
$$

where $q$ is the coefficient, which is given by Kim's model as

$$
q= \begin{cases}1.6, & \text { if } V>50 \mathrm{~km}, \\ 1.3, & \text { if } 6 \mathrm{~km}<V \leq 50 \mathrm{~km}, \\ 0.16 V+0.34, & \text { if } 1 \mathrm{~km}<V \leq 6 \mathrm{~km}, \\ V-0.5, & \text { if } 0.5 \mathrm{~km}<V \leq 1 \mathrm{~km}, \\ 0, & \text { if } V \leq 0.5 \mathrm{~km} .\end{cases}
$$

Turbulence-induced fading: The Gamma-Gamma distribution is used for modeling the turbulence from weak regime to strong regime. The PDF of the turbulence coefficient can be expressed

$$
f_{h_{a}}\left(h_{a}\right)=\frac{2(\alpha \beta)^{\frac{\alpha+\beta}{2}}}{\Gamma(\alpha) \Gamma(\beta)} h_{a}^{\frac{\alpha+\beta}{2}} K_{\alpha-\beta}\left(2 \sqrt{\alpha \beta h_{a}}\right),
$$

where $\Gamma(\cdot)$ is the gamma function and $K_{v}(\cdot)$ is the $v$-th order modified Bessel function of the second kind. In addition, $\alpha$ and $\beta$ can be found in [29], which is the function of Rytov variance, $\sigma_{R}^{2}$, and for the plane wave, $\sigma_{R}^{2}$ can be given as

$$
\sigma_{R}^{2}=2.25 k_{w}^{7 / 6} \sec ^{11 / 6}\left(\xi_{p}\right) \int_{H_{u}}^{H_{p}} C_{n}^{2}(h)\left(h-H_{u}\right)^{5 / 6} d h,
$$

where $k_{w}=2 \pi / \lambda$ is the optical wave number. Based on Hufnagel-Valley model [29], the variation of refractive index structure parameter $C_{n}^{2}(h)$ according to the altitude $h$ can be written as

$$
\begin{gathered}
C_{n}^{2}(h)=0.00594\left(\frac{v_{w i n d}}{27}\right)^{2}\left(10^{-5} h\right) \exp \left(-\frac{h}{1000}\right) \\
+2.7 \times 10^{-16} \exp \left(-\frac{h}{1500}\right)+C_{n}^{2}(0) \exp \left(-\frac{h}{100}\right),
\end{gathered}
$$

where $C_{n}^{2}(0)$ is the ground turbulence level varying in the range of $10^{-17} \mathrm{~m}^{-2 / 3}$ (weak turbulence regime) to $10^{-13} \mathrm{~m}^{-2 / 3}$ (strong turbulence regime), and $v_{\text {wind }}(\mathrm{m} / \mathrm{s})$ is the root mean squared wind speed with typical value of 21 $\mathrm{m} / \mathrm{s}$ [29].

UAV hovering misalignment model: Pointing error is mainly caused by the wind pushing the UAV around its operating position. The HAP, on the contrary, is assumed to remain stable as it is located at a much higher altitude where there is little wind. The hovering results in a change of position of the UAV aperture. Note that the UAV's position is not necessarily in the center of the beam footprint for the purpose of serving multiple UAVs at the same time. Let $r$ be the radial vector from the beam center to the UAV's initial position. Due to the wind, UAV hovers around $r_{h}$ radial to another position. Consequently, the actual position can be a $r_{p e}$ radial

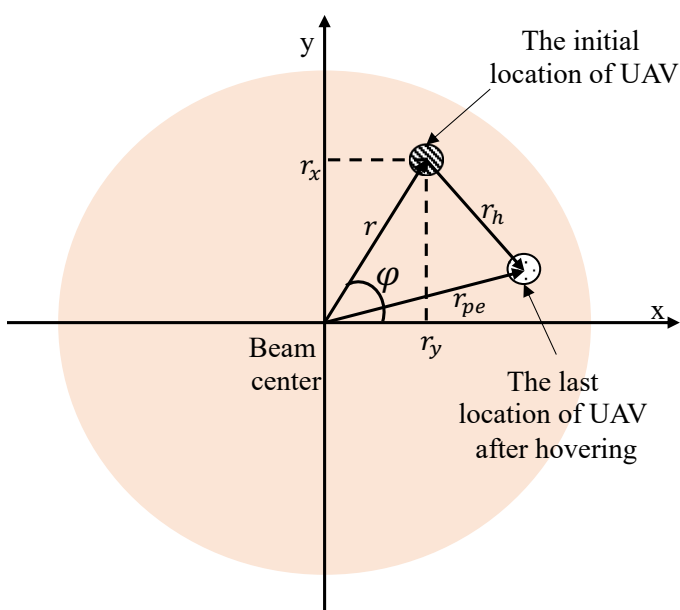

Fig. 4. UAV hovering misalignment model.

distance from the beam footprint, and it can be expressed in the coordinate plane as followed

$r_{p e}\left(x_{r_{p e}}, y_{r_{p e}}\right)=\left\{\begin{array}{l}x_{r_{p e}}=r_{x}+x_{r_{h}}=\|r\| \sin (\varphi)+x_{r_{h}}, \\ y_{r_{p e}}=r_{y}+y_{r_{h}}=\|r\| \cos (\varphi)+y_{r_{h}},\end{array}\right.$

where $\|\cdot\|$ is the norm of a vector, $x_{r_{h}}$ and $y_{r_{h}}$ are zero-mean Gaussian random variables with variance $\sigma_{p}^{2}$. Besides, $x_{r_{p e}}$ and $y_{r_{p e}}$ are two statistically independent Gaussian random variables as $x_{r_{p e}} \sim \mathcal{N}\left(r_{x}, \sigma_{p}^{2}\right)$ and $y_{r_{p e}} \sim \mathcal{N}\left(r_{y}, \sigma_{p}^{2}\right)$. As reported in [34], the variance of $x_{r_{p e}}$ and $y_{r_{p e}}$ are the same and equal $\sigma_{p}^{2}$, the PDF of radial displacement $r_{p e}$ follows Rician distribution

$$
f_{r_{p e}}\left(r_{p e}\right)=\frac{r_{p e}}{\sigma_{p}^{2}} \exp \left[-\frac{\left(r_{p e}^{2}+r^{2}\right)}{2 \sigma_{p}^{2}}\right] I_{0}\left(\frac{r_{p e} r}{\sigma_{p}^{2}}\right)
$$

where $I_{0}(\cdot)$ is the modified Bessel function of the first kind with order zero, $\sigma_{p e}^{2}$ is the jitter variance. By considering the Gaussian beam, the UAV hovering misalignment coefficient can be expressed as

$$
h_{p e}=B_{0} \exp \left(-\frac{2 r_{p e}^{2}}{w_{z e q}^{2}}\right)
$$

where $B_{0}=\left[\operatorname{erf}\left(v_{2}\right)\right]^{2}$ is the fraction of the collected power by UAV' $\mathrm{s}$ detector at $r_{p e}=0 . w_{z e q}^{2}=\frac{\sqrt{\pi} \operatorname{erf}\left(v_{2}\right)}{2 v_{2} \exp \left(-v_{2}^{2}\right)} w_{z}^{2}$ is the equivalent beam-width, $v_{2}=\frac{a_{2} \sqrt{\pi}}{w_{z} \sqrt{2}}$ in which $a_{2}$ is the radius of detector, $w_{z} \approx w_{z 0} \sqrt{1+\varepsilon_{2}\left(\frac{\lambda d_{2}}{\pi w_{z 0}^{2}}\right)^{2}}$ is the beam waist at the distance $d_{2}, w_{z 0}$ is the beam waist at $d_{2}=0$, $\varepsilon_{2}=\left(1+2 \frac{w_{z 0}^{2}}{\rho_{z 0}^{2}\left(d_{2}\right)}\right)$, and $\rho_{z 0}\left(d_{2}\right)=\left(0.55 C_{n}^{2} k_{w}^{2} d_{2}\right)^{-3 / 5}$ is the coherence length. As derived in [34], [35], the PDF of 
pointing error $h_{p e}$ can be expressed as

$$
\begin{aligned}
f_{h_{p}}\left(h_{p}\right) & =\frac{w_{d e q}^{2}}{4 A_{0} \sigma_{p}^{2}} \exp \left(-\frac{r^{2}}{2 \sigma_{p}^{2}}\right)\left(\frac{h_{p}}{A_{0}}\right)^{\frac{w_{d e q}^{2}}{4 \sigma_{p}^{2}}} \\
& \times I_{0}\left(\frac{r}{\sigma_{p}^{2}} \sqrt{-\frac{w_{d e q}^{2}}{2} \ln \left(\frac{h_{p}}{A_{0}}\right)}\right),
\end{aligned}
$$

Joint PDF and CDF of combined channel: After mathematical manipulation, the joint distribution of the channel coefficient $h_{F S O}$ can be expressed as

$$
\begin{aligned}
f_{h_{F S O}} & \left(h_{F S O}\right)=\sum_{i=1}^{n} 4 x_{i} w_{i} \frac{(\alpha \beta)^{\frac{\alpha+\beta}{2}}}{\Gamma(\alpha) \Gamma(\beta)} \exp \left(-\frac{r^{2}}{4 \sigma_{p}^{2}}\right) \\
& \times\left(\frac{1}{A_{0} h_{c}}\right)^{\frac{\alpha+\beta}{2}} \exp \left[\frac{4 x_{i}^{2} \sigma_{p}^{2}(\alpha+\beta)}{w_{z e q}^{2}}\right] I_{0}\left(\frac{r x_{i}}{\sigma_{p}}\right) \\
& \times K_{\alpha-\beta}\left(2 \sqrt{\frac{\alpha \beta h_{F S O}}{A_{0} h_{c}} \exp \left(\frac{8 x_{i}^{2} \sigma_{p}^{2}}{w_{z e q}^{2}}\right)}\right) h_{F S O}^{\frac{\alpha+\beta}{2}-1} .
\end{aligned}
$$

where $x_{i}$ and $w_{i}$ are the weights and the zeros of the Hermite polynomial, respectively. As reported in [34], the PDF of received SNR of FSO channel from HAP to UAV can be expressed

$$
\begin{aligned}
& f_{\gamma_{2, F S O}}\left(\gamma_{2, F S O}\right)=\sum_{i=1}^{n} 2 x_{i} w_{i} \frac{(\alpha \beta)^{\frac{\alpha+\beta}{2}}}{\Gamma(\alpha) \Gamma(\beta)} \exp \left(-\frac{r^{2}}{2 \sigma_{p}^{2}}\right) k_{p e^{\frac{\alpha+\beta}{2}}} \\
& \times \exp \left[\frac{2 x_{i}^{2} \sigma_{p}^{2}(\alpha+\beta)}{w_{L e q}^{2}}\right] I_{0}\left(\frac{r \sqrt{2} x_{i}}{\sigma_{p}}\right)\left(\frac{1}{\bar{\gamma}_{2, F S O}}\right)^{\frac{\alpha+\beta}{4}} \\
& \times K_{\alpha-\beta}\left(2 \sqrt{\left.\alpha \beta k_{p e} \sqrt{\frac{\gamma_{2, F S O}}{\bar{\gamma}_{2, F S O}}} \exp \left(\frac{4 x_{i}^{2} \sigma_{p}^{2}}{w_{L e q}^{2}}\right)\right) \gamma_{2, F S O}^{\frac{\alpha+\beta}{4}}-1} .\right. \text { (26) }
\end{aligned}
$$

By applying [36, Eq.(8.4.23)], [37, Eq. (26)], and after several mathematical manipulations, the CDF of the SNR of FSO link can be shown as in Eq. (27), in which the value of $k_{p e}$ can be found in [34].

\section{RATE ADAPTATION DESIGN}

This section focuses on the design of rate adaptation for our proposed systems. Several performance metrics are then derived in our analysis, including outage probability, average transmission rate, achievable spectrum efficiency, and average bit error rate.

\section{A. Adaptive Transmission Schemes}

The purpose of adaptive multi-rate is to provide as high data-rate as possible depending on channel conditions while maintaining a required quality of service $(\mathrm{QoS})$. This proposed system aims to transmit the highest data rate in the FSO link and the lower data rate in the RF link, and the outage occurs if the channel condition is very poor. To support rate adaptation, the channel estimator is designed at the destination to estimate the FSO and RF channel state information (CSI) as shown in Fig. 2. The CSI reflects the information regarding the selected link and transmission rate information depending on the received SNR of the FSO and RF links. The logical channel is assumed error-free channel to feedback CSI, and the information is then sent back to the relay node (i.e., HAP's link switching controller) and source node (i.e., satellite's rateadaptive controller). Depending on the CSI, HAP's switching controller decides which link is transmitted on the next stage and which transmission rate is determined by the satellite's rate-adaptive controller.

Table I introduces the generalized ${ }^{1}$ design of rate adaptation with QAM constellation. There are three transmission modes, including FSO, RF, and outage, in which FSO and RF have several different transmission rate sub-modes. Depending on the transmission environment, the FSO link and RF link are switched to each other. If the channel quality is not good, the transmission rate of the FSO link will gradually degrade from the left to the right side, correspond to reduce the modulation order of the QAM constellation. If the channel condition continuously worsens, the FSO link will off, and the $\mathrm{RF}$ link will be activated to maintain the communications. The transmission rate mode of the RF link is also designed to adapt to environmental conditions. It means that the transmission rate will decrease, stay in the current mode or increase respective to the channel quality become poor, unchanged, or better.

To support multi-rate for each link, we employ adaptive modulation. The QAM is suitable for both links in high-speed vertical communications due to its support for higher-order modulation. The other modulations, such as OOK and PPM, do not support higher-order modulation and cannot support transmitting high data rates. The transmission rate is given as

$$
R=\left\{\begin{array}{lll}
R_{f} \log _{2} M, & \text { if } \gamma_{f(j)} \leq \gamma_{f}<\gamma_{f(j+1)}, & \text { FSO link } \\
R_{r} \log _{2} N, & \text { if } \gamma_{r(i)} \leq \gamma_{r}<\gamma_{r(i+1)}, & \text { RF link } \\
0, & \text { if } 0 \leq \gamma_{r}<\gamma_{r(1)}, & \text { Outage }
\end{array}\right.
$$

where $R_{f}$ and $R_{r}$ are the symbol rate of the FSO link and RF link, respectively. As note of the table I, transmission rate is associated with the SNR region and the order-modulation $M$ or $N$ of FSO link or RF link, in which $M=2^{m}(i=$ $1,2, \ldots, m), N=2^{n}(j=1,2, \ldots, n)$ and $m, n$ are the number of bit per QAM symbol. A generalized design of adaptive multi-rate with QAM constellation is depicted in Table I. For each transmission mode, we divide each FSO or RF mode into multiple sub-mode. In physical layer, those sub-modes are defined to satisfy a requirement of QoS such as the target bit error rate $\left(\mathrm{BER}_{0}\right)$. As reported in [38], the $\mathrm{BER}_{0}$ of $M$ QAM over the AWGN channel can be expressed

$$
\operatorname{BER}_{0}(M, \gamma) \cong 0.2 \exp \left[-\frac{3 \gamma}{2(M-1)}\right]
$$

The switching threshold is derived as

$$
\gamma=\frac{-2(M-1)}{3} \ln \left[5 \operatorname{BER}_{0}(M, \gamma)\right]
$$

\footnotetext{
${ }^{1}$ This study uses QAM modulations to support high-speed connections. The design of rate adaptation can also be applied to other modulation schemes, e.g., ASK, PSK, and PAM, etc.
} 


$$
\begin{aligned}
F_{\gamma_{2, F S O}}\left(\gamma_{2, F S O}\right) & =\sum_{i=1}^{n} 2 x_{i} w_{i} \frac{(\alpha \beta)^{\frac{\alpha+\beta}{2}}}{\Gamma(\alpha) \Gamma(\beta)} \exp \left(-\frac{r^{2}}{2 \sigma_{p}^{2}}\right) k_{p e}^{\frac{\alpha+\beta}{2}} \exp \left[\frac{2 x_{i}^{2} \sigma_{p}^{2}(\alpha+\beta)}{w_{L e q}^{2}}\right] I_{0}\left(\frac{r \sqrt{2} x_{i}}{\sigma_{p}}\right)\left(\frac{\gamma_{2, F S O}}{\bar{\gamma}_{2, F S O}}\right)^{\frac{\alpha+\beta}{4}} \\
& \times G_{1,3}^{2,1}\left[\alpha \beta k_{p e} \exp \left(\frac{4 x_{i}^{2} \sigma_{p}^{2}}{w_{L e q}^{2}}\right) \sqrt{\frac{\gamma_{2, F S O}}{\bar{\gamma}_{2, F S O}}} \mid \frac{\alpha-\beta}{2}, \frac{-\frac{\alpha+\beta}{2}}{2},-\frac{\alpha+\beta}{2}\right] .
\end{aligned}
$$

TABLE I

\begin{tabular}{|c|c|c|c|c|c|c|c|c|c|c|}
\hline \multirow{2}{*}{\multicolumn{2}{|c|}{$\begin{array}{l}\text { Transmission mode } \\
\text { Transmission Rate }\end{array}$}} & \multicolumn{4}{|c|}{ FSO } & \multicolumn{4}{|c|}{$\mathrm{RF}$} & \multirow{2}{*}{$\begin{array}{c}\text { Outage } \\
0\end{array}$} \\
\hline & & $m R_{f}$ & $\ldots$ & $(i+1) R_{f}$ & $i R_{f}$ & $n R_{r}$ & $\ldots$ & $(j+1) R_{r}$ & $j R_{r}$ & \\
\hline SNR & FSO & {$\left[\gamma_{f(m)}, \infty\right)$} & $\ldots$ & {$\left[\gamma_{f(i+1)}, \ldots\right)$} & {$\left[\gamma_{f(i+1)}, \gamma_{f(i+2)}\right)$} & \multicolumn{5}{|c|}{$\left[0, \gamma_{f(i)}\right)$} \\
\hline conditions & $\mathrm{RF}$ & \multicolumn{4}{|c|}{ No consideration } & {$\left[\gamma_{r(n)}, \infty\right)$} & $\ldots$ & {$\left[\gamma_{f(j+1)}, \ldots\right)$} & {$\left[\gamma_{r(j+1)}, \gamma_{r(j+2)}\right)$} & {$\left[0, \gamma_{r(j)}\right)$} \\
\hline \multicolumn{2}{|c|}{ Constellation size } & $2^{m}$ & $\ldots$ & $2^{i+1}$ & $2^{i}$ & $2^{n}$ & $\ldots$ & $2^{j+1}$ & $2^{j}$ & No transmission \\
\hline
\end{tabular}

A GENERALIZED DESIGN OF RATE ADAPTATION WITH QAM CONSTELLATION.

\section{B. Performance Analysis}

1) Outage probability: The outage occurs when the system moves to the zero-rate mode, in which no data is transmitted to avoid the high error rate. The outage probability is then calculated as

$$
\begin{aligned}
P_{\text {out }} & =\int_{0}^{\gamma_{e 2 e}^{f(1)}} f_{\gamma_{e 2 e}^{f}}\left(\gamma_{e 2 e}^{f}\right) d \gamma_{e 2 e}^{f} \int_{0}^{\gamma_{e 2 e}^{r(1)}} f_{\gamma_{e 2 e}^{r}}\left(\gamma_{e 2 e}^{r}\right) d \gamma_{e 2 e}^{r} \\
& =F_{\gamma_{e 2 e}^{f}}\left(\gamma_{e 2 e}^{f}\right) F_{\gamma_{e 2 e}^{r}}\left(\gamma_{e 2 e}^{r}\right),
\end{aligned}
$$

where $F_{\gamma_{e 2 e}^{r}}\left(\gamma_{e 2 e}^{r}\right)$ and $F_{\gamma_{e 2 e}^{f}}\left(\gamma_{e 2 e}^{f}\right)$ are respectively the CDFs of end-to-end for the dual-hop of FSO-RF and FSO-FSO, which are expressed as

For FSO-RF link:

$$
F_{\gamma_{e 2 e}^{r}}\left(\gamma_{e 2 e}^{r}\right)=\operatorname{Pr}\left(\frac{\gamma_{1} \gamma_{2, \mathrm{RF}}}{\gamma_{2, \mathrm{RF}}+1}<\gamma_{e 2 e}^{r}\right)=F_{\gamma_{2, \mathrm{RF}}}\left(\frac{\gamma_{e 2 e}^{r}}{\gamma_{1}-\gamma_{e 2 e}^{r}}\right) \text {. }
$$

\section{For FSO-FSO link:}

$F_{\gamma_{e 2 e}^{f}}\left(\gamma_{e 2 e}^{f}\right)=\operatorname{Pr}\left(\frac{\gamma_{1} \gamma_{2, \mathrm{FSO}}}{\gamma_{2, \mathrm{FSO}}+1}<\gamma_{e 2 e}^{f}\right)=F_{\gamma_{2, \mathrm{FSO}}}\left(\frac{\gamma_{e 2 e}^{f}}{\gamma_{1}-\gamma_{e 2 e}^{f}}\right)$.

2) Average transmission rate: The average transmission rate, which is an essential parameter in adaptive multi-rate systems, can be determined as

$$
\bar{R}=\sum_{k=1}^{N} p_{k} R_{k}
$$

where $N$ is the total modes. Since the transmission rate of each link is fixed, the link switching threshold is designed based on targeted BER, then the fixed $M$-QAM is used. Mode probability is defined as the probability that k-th mode is selected; then it can be calculated as

\section{For FSO-RF link}

$$
\begin{aligned}
& p_{k}^{R F}=\int_{0}^{\gamma_{e 2 e}^{f(1)}} f_{\gamma_{e 2 e}^{f}}\left(\gamma_{e 2 e}^{f}\right) d \gamma_{e 2 e}^{f} \int_{\substack{\gamma_{e 2 e}^{r(j)} \\
\gamma_{e 2 e}^{r(j+1)}}}^{\gamma_{\gamma_{e 2 e}^{r}}^{r}\left(\gamma_{e 2 e}^{r}\right) d \gamma_{e 2 e}^{r}} \\
& =\left[1-F_{\gamma_{e 2 e}^{f}}\left(\gamma_{e 2 e}^{f(1)}\right)\right]\left[F_{\gamma_{e 2 e}^{r}}\left(\gamma_{e 2 e}^{r(j+1)}\right)-F_{\gamma_{e 2 e}^{r}}\left(\gamma_{e 2 e}^{r(j)}\right)\right] .
\end{aligned}
$$

\section{For FSO-FSO link}

$$
\begin{aligned}
p_{k}^{F S O} & =\int_{\gamma_{e 2 e}^{f(i)}}^{\gamma_{e 2 e}^{f(i+1)}} f_{\gamma_{e 2 e}^{f}}\left(\gamma_{e 2 e}^{f}\right) d \gamma_{e 2 e}^{f} \\
& =F_{\gamma_{e 2 e}^{f}}\left(\gamma_{e 2 e}^{f(i+1)}\right)-F_{\gamma_{e 2 e}^{f}}\left(\gamma_{e 2 e}^{f(i)}\right) .
\end{aligned}
$$

3) Spectrum efficiency: As the system is in the state $k$ th, each transmitted symbol carries $R_{k}$ information bits. We suppose that a Nyquist pulse shaping filter with the bandwidth $B=1 / R_{0}$. Then, the achievable spectral efficiency, defined as the data rate per unit bandwidth, is given by

$$
S_{\text {eff }}=\frac{1}{R_{0}} \sum_{k=1}^{N} p_{k} R_{k}
$$

4) Average bit error rate (ABER): The ABER is defined as the ratio of the average number of erroneously received bits over the total average number of transmitted bits, which is expressed by

$$
\mathrm{ABER}=\frac{\text { Total errorneous bits }}{\text { Total transmitted bits }}=\frac{\sum_{k=1}^{N} R_{k} p_{k} \mathrm{BER}_{k}}{\sum_{k=1}^{N} R_{k} p_{k}},
$$

where $R_{k}$ is the transmission rate at state $k$-th, $\mathrm{BER}_{k}$ is the bit error rate of selecting mode $k$-th over fading channel, which can be determined as

\section{For FSO-FSO link}

$$
\operatorname{BER}_{f s o}=\frac{1}{p_{k}^{F S O}} \int_{\gamma_{f(k)}}^{\gamma_{f(k+1)}} \operatorname{BER}\left(M, \gamma_{f}\right) f_{\gamma_{f}}\left(\gamma_{f}\right) d \gamma_{f} .
$$

\section{For FSO-RF link}

$$
\operatorname{BER}_{r f}=\frac{1}{p_{k}^{R F}} \int_{\gamma_{f(k)}}^{\gamma_{f(k+1)}} \operatorname{BER}\left(M, \gamma_{r}\right) f_{\gamma_{r}}\left(\gamma_{r}\right) d \gamma_{r}
$$




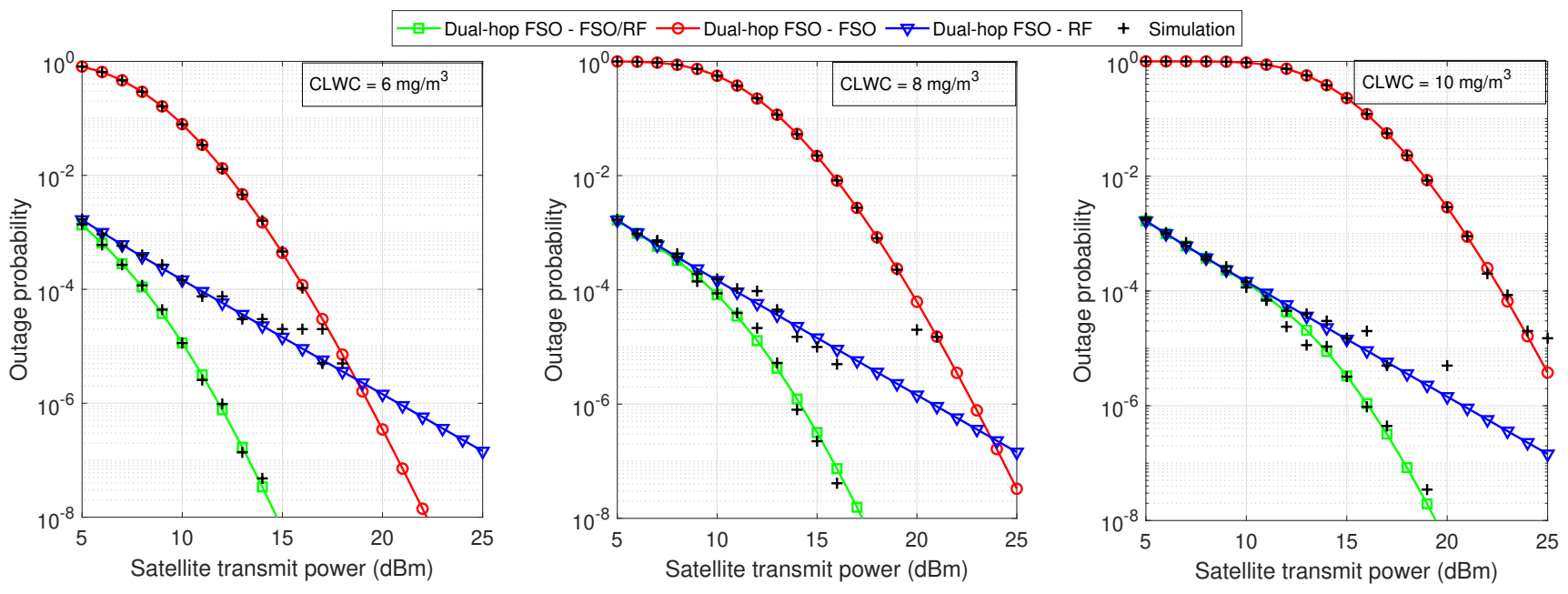

Fig. 5. Effects of clouds on the outage performance with respect to the distance from UAV to beam center of $20 \mathrm{~m}$.

\section{Numerical Results}

In this section, we present selected numerical results to demonstrate the advantages of our proposed adaptive hybrid FSO/RF design in the scenario of space-air-ground networks. Notably, we highlight the effectiveness of our proposed systems by comparing performance metrics analyzed in Section IV with those of conventional systems, i.e., adaptive dual-hop FSO-FSO, adaptive dual-hop FSO-RF, and fixedrate dual-hop FSO-hybrid FSO/RF designs. To this purpose, the sets of transmission modes using $M$-QAM modulation with $M \in\{2,4,8,16,32,64,128\}$ for FSO links and $N$ QAM modulation with $N \in\{2,4,8,16,32\}$ for RF links ${ }^{2}$ are adopted for the adaptive multi-rate design. For the sake of comparison, 32-QAM modulation is given for RF links, while different $M$-QAM modulations are considered for FSO links, in the conventional fixed-rate systems. The parameters used in the analysis, unless otherwise noted, are given in Table II. Moreover, the Monte Carlo simulations using MATLAB programing are also performed to validate the analytical results.

We first analyze the effectiveness of our proposed dualhop FSO-hybrid FSO/RF adaptive systems by comparing with the conventional adaptive ones, including dual-hop FSO-FSO and dual-hop FSO-RF, in terms of outage performance, over a range of satellite's transmitted power in Fig. 5. Here, different cloud conditions, i.e., CLWC $=6 \mathrm{mg} / \mathrm{m}^{3}$, CLWC $=8 \mathrm{mg} / \mathrm{m}^{3}$, and CLWC $=10 \mathrm{mg} / \mathrm{m}^{3}$, which are the critical concerns, especially for FSO links, are investigated. There are two remarkable observations here. Firstly, when the CLWC increases, the outage performance of dual-hop FSO-FSO systems is significantly deteriorated, while a minimal change is witnessed for dual-hop FSO-RF ones. The reason is that the impact of clouds is much more severe in FSO systems as the optical wavelength is smaller than the typical cloud droplet size values [23]. Contrary to optical bands, the wavelengths of RF ones

\footnotetext{
${ }^{2}$ It is worth noting that more transmission modes result in better performance while the system complexity is increased. As FSO is the primary link, the number of RF modes is, therefore, selected to be less than the FSO ones.
}

TABLE II

SYSTEM PARAMETERS

\begin{tabular}{|c|c|c|}
\hline Name & Symbol & Value \\
\hline \multicolumn{3}{|c|}{ LEO Satellite } \\
\hline LEO satellite altitude & $H_{s}$ & $530 \mathrm{~km}$ \\
\hline Divergence angle & $\theta_{\text {sat }}$ & $15 \mu \mathrm{rad}$ \\
\hline Optical wavelength & $\lambda$ & $1550 \mathrm{~nm}$ \\
\hline RF frequency & $f_{r}$ & $2 \mathrm{GHz}$ \\
\hline FSO symbol rate & $R_{f}$ & $500 \mathrm{Msps}$ \\
\hline RF symbol rate & $R_{r}$ & 100 Msps \\
\hline \multicolumn{3}{|c|}{ High Altitude Platform (HAP) } \\
\hline HAP altitude & $H_{p}$ & $20 \mathrm{~km}$ \\
\hline HAP aperture radius & $r_{\text {hap }}$ & $10 \mathrm{~cm}$ \\
\hline Zenith angle & $\xi_{p}$ & $60^{\circ}$ \\
\hline Divergence angle & $\theta_{\text {hap }}$ & $1.5 \mathrm{mrad}$ \\
\hline \multicolumn{3}{|c|}{ Unnamed Aerial Vehicle (UAV) } \\
\hline UAV altitude & $H_{u}$ & $100 \mathrm{~m}$ \\
\hline Hovering jitter variance & $\sigma_{p}$ & $0.9 \mathrm{~m}$ \\
\hline UAV aperture radius & $r_{u a v}$ & $5 \mathrm{~cm}$ \\
\hline Detector responsivity & $\mathcal{R}$ & 0.9 \\
\hline Noise standard deviation & $\sigma_{n}$ & $10^{-7} \mathrm{~A} / \mathrm{Hz}$ \\
\hline Gaseous atmosphere losses & $L_{A}$ & $5.4 \times 10^{-3} \mathrm{~dB} / \mathrm{km}$ \\
\hline Receiver noise bandwidth & $B W$ & $74.8 \mathrm{dBHz}$ \\
\hline System noise temperature & $T_{N}$ & $17.6 \mathrm{dBK}$ \\
\hline Noise figure & $N_{F}$ & $2 \mathrm{~dB}$ \\
\hline \multicolumn{3}{|c|}{ Other Parameters } \\
\hline Targeted BER & $\mathrm{BER}_{0}$ & $10^{-5}$ \\
\hline Number of cloud concentrations & $N_{c}$ & $250 \mathrm{~cm}^{-3}$ \\
\hline Considered length of clouds & $d_{z}$ & $2 \mathrm{~km}$ \\
\hline Turbulence at ground level & $C_{n}^{2}(0)$ & $10^{-13} \mathrm{~m}^{-2 / 3}$ \\
\hline Rician factor & $K$ & $6 \mathrm{~dB}$ \\
\hline
\end{tabular}

are often larger than the size of cloud droplets, resulting in a very low attenuation caused by clouds on such links [26]. In addition, with high values of the satellite's transmitted power, the dual-hop FSO-FSO systems, however, can maintain a better performance than dual-hop FSO-RF ones in less severe cloud conditions, e.g., CLWC $=6 \mathrm{mg} / \mathrm{m}^{3}$ and CLWC $=8$ $\mathrm{mg} / \mathrm{m}^{3}$. Secondly, as is expected, our proposed dual-hop FSOhybrid FSO/RF systems can provide a significant performance improvement over the conventional ones in different cloud conditions, thanks to inheriting both FSO and RF benefits. Also, in this figure, the analytical results follow the simulated 

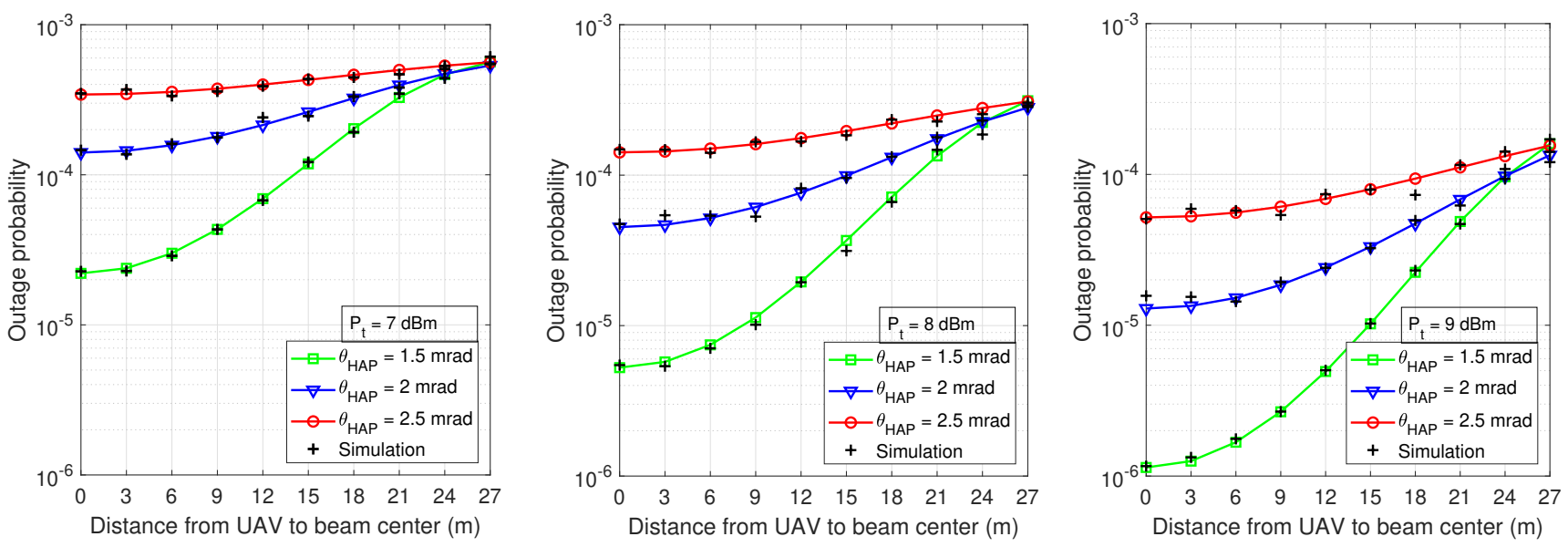

Fig. 6. Outage probability versus satellite transmit power and distance from center of Gaussian beam to UAV position with CLWC of $6 \mathrm{mg} / \mathrm{m}^{3}$.

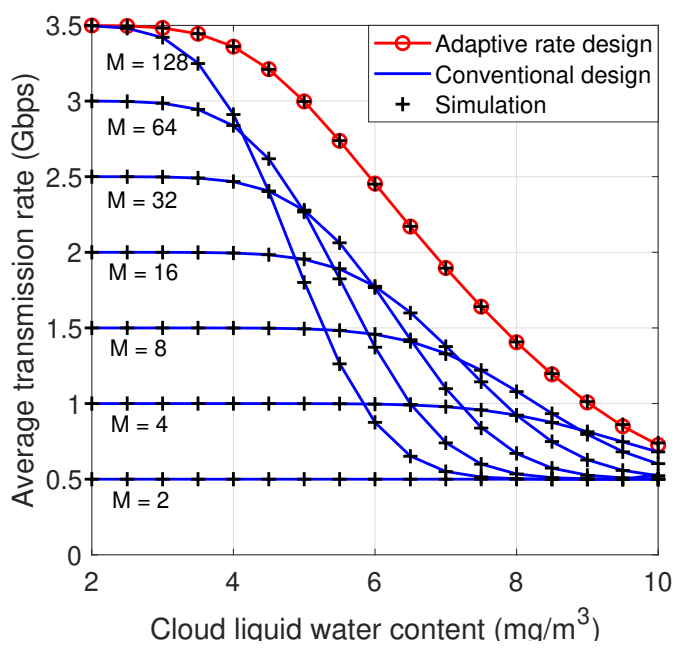

Fig. 7. The impact of cloud to average transmission rate of adaptive rate system and fixed rate system.

ones very closely, which confirms the correctness of the model and analysis.

In Fig. 6, we investigate the outage performance in terms of beam footprint size provided by the HAP-based relaying node, when CLWC $=6 \mathrm{mg} / \mathrm{m}^{3}$. Also, different satellite's transmitted powers, i.e., $P_{\mathrm{t}}=7 \mathrm{dBm}, 8 \mathrm{dBm}$, and $9 \mathrm{dBm}$, and HAP's divergence angles, i.e., $\theta_{\mathrm{HAP}}=1.5 \mathrm{mrad}, 2 \mathrm{mrad}$, and $2.5 \mathrm{mrad}$, are taken into account. This is a vital performance metric to determine the system operational coverage area. For instance, when $\theta_{\mathrm{HAP}}=1.5 \mathrm{mrad}$, to retain a target outage performance level of below $10^{-4}$, the operational area of UAVs should be within $15 \mathrm{~m}, 19 \mathrm{~m}, 24 \mathrm{~m}$, for $P_{\mathrm{t}}=7 \mathrm{dBm}, 8 \mathrm{dBm}$, and 9 $\mathrm{dBm}$, respectively. In addition, the highly directional beam is obtained for smaller HAP's divergence angles, resulting in a better outage performance. Such smaller HAP's divergence angles, nevertheless, restrict the system operational areas, leading to the high outage probability at the edge of the beam footprint. As a result, there exists a tradeoff between

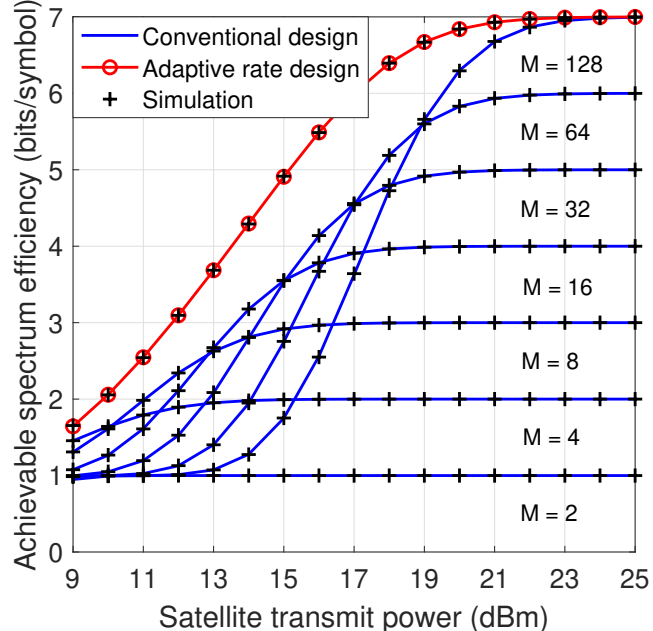

Fig. 8. The achievable spectrum efficiency versus satellite transmit power in the case of adaptive rate system and fixed rate system.

the operational areas and system performance in the selection of HAP's divergence angles.

Next, we further analyze the advantages of our proposed adaptive multi-rate design in comparison with the conventional systems based fixed-rate schemes. Specifically, Fig. 7 investigates the achievable data rate over a range of CLWCs, when $P_{\mathrm{t}}=15 \mathrm{dBm}$. Obviously, compared to the fixed-rate scheme, the adaptive multi-rate design could offer considerably better performance under the impact of cloud coverage. This fact can be explained from two remarkable points: (i) the systems with high modulation size could maintain a high maximum achievable data rate in less severe cloud conditions; and (ii) the performance of such systems using high modulation size is significantly degraded in more severe cloud conditions, in which low modulation size is preferable in this situation, leading to the limitation of maximum achievable data rate. As a result, systems using a fixed-rate scheme may not be efficient in time-varying channels, and adaptive multi-rate design would 

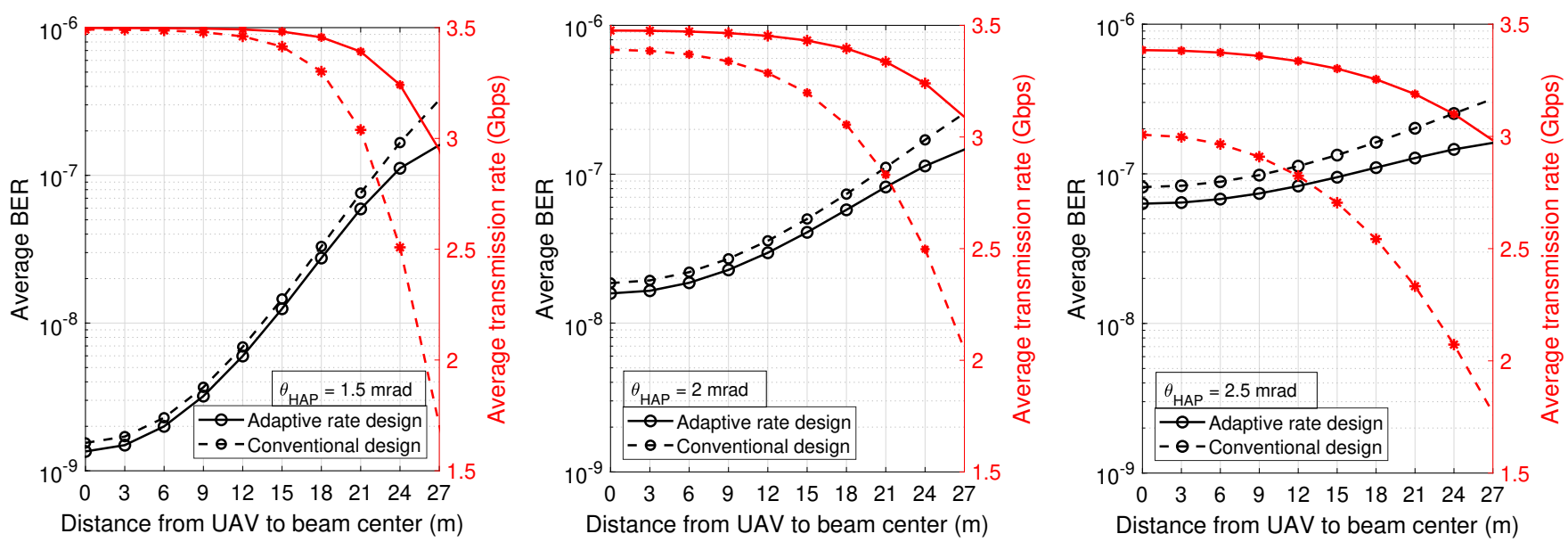

Fig. 9. Impact of UAV location to Average BER for different value of HAP divergence angle with satellite transmit power of $20 \mathrm{dBm}$.

be the promising solution. For example, when CLWC $=6$ $\mathrm{mg} / \mathrm{m}^{3}$, our proposed adaptive system is able to achieve 2.45 Gbps of data rate, while the fixed-rate one could only retain the data rate of $1.77 \mathrm{Gbps}, 1.45 \mathrm{Gbps}, 1.37 \mathrm{Gbps}, 0.99 \mathrm{Gbps}$, and 0.5 Gbps with 32-QAM, 8-QAM, 64-QAM, 128-QAM, 2-QAM modulations used for the FSO links, respectively. The outperformance of the proposed adaptive multi-rate design over the conventional fixed-rate one can also be observed in Fig. 8 in which variations in achievable spectrum efficiency performance with different values of satellite's transmitted power, when CLWC $=6 \mathrm{mg} / \mathrm{m}^{3}$. For instance, when $P_{\mathrm{t}}=20$ $\mathrm{dBm}$, our proposed design can achieve the spectrum efficiency of $6.8 \mathrm{bits} / \mathrm{symbol}$, while they are only $6.2 \mathrm{bits} / \mathrm{symbol}, 5.8$ bits/symbol, 4.9 bits/symbol, 4 bits/symbol, 3 bits/symbol, 2 bits/symbol, 1 bits/symbol for respectively 128-QAM, 64QAM, 32-QAM, 16-QAM, 8-QAM, 4-QAM, 2-QAM with the fixed-rate one.

Finally, we highlight the effectiveness of our proposed adaptive design by comparing the average BER performance with that of conventional fixed-rate one, over a range of beam footprint sizes, as illustrated in Fig. 9. Also, $P_{\mathrm{t}}=20 \mathrm{dBm}$, CLWC $=6 \mathrm{mg} / \mathrm{m}^{3}$, and different HAP's divergence angles, i.e., $\theta_{\mathrm{HAP}}=1.5 \mathrm{mrad}, 2 \mathrm{mrad}$, and $2.5 \mathrm{mrad}$, are considered. As expected, our proposed design offers a lower average BER performance compared to the conventional one, even with higher achievable data-rate transmissions. This phenomenon is due to the fact that the objective of adaptive multi-rate design is to maximize the data rate over the time-varying turbulence-induced fading channels while satisfying a predefined Quality of Service (QoS), i.e., a targeted BER. Moreover, using this figure, we can decide the operational range of UAVs to maintain a targeted BER performance by selecting a proper HAP's divergence angle parameter, which is especially essential parameter for mobile users. For example, to retain a level of BER performance of below $10^{-7}$, the operational area of UAVs should be within $24 \mathrm{~m}, 22 \mathrm{~m}$, and $17 \mathrm{~m}$ for $\theta_{\mathrm{HAP}}=$ $1.5 \mathrm{mrad}, 2 \mathrm{mrad}$, and $2.5 \mathrm{mrad}$, respectively.

\section{CONCluding Remarks And Future WORK}

We have proposed the comprehensive adaptive hybrid FSO/RF design and analytical framework for the performance of HAP-aided relaying satellite communications for mobile networks supported by UAVs. For hybrid FSO/RF channels, the Rician distribution was adopted for RF links, while several factors were taken into account on FSO links, including atmospheric turbulence modeled by the Gamma-Gamma distribution, the cloud coverage, and the UAV hovering-induced pointing misalignment. Several system performance metrics, including outage probability, average transmission rate, achievable spectrum efficiency, and average bit error rate, were analytically derived.

Through the numerical results, there are several concluding remarks: (i) Our proposed system could offer a significant performance improvement compared to the existing designs in the scenario of integrated downlink satellite-HAPground/vehicles, i.e., adaptive design (dual-hop FSO-FSO and dual-hop RF-RF) and fixed-rate design (dual-hop FSO-hybrid FSO/RF); (ii) Our results could support the proper parameter selection for practical mobile users, i.e., beam size provided by HAP and satellite's transmitted power; and (iii) the correctness of our analytical model was confirmed by the Monte Carlo simulations, in which the excellent agreement between theoretical results and simulated ones were validated.

Our study could be a generalized framework for relay-based satellite-assisted vehicular networks, not only with UAVs but also for other vehicles, e.g., high-speed trains and autonomous cars. For future work, it would be interesting to investigate other adaptive schemes, i.e., power adaptation, which is especially essential to save energy consumption in the domain of satellite and/or vehicular networks.

\section{REFERENCES}

[1] "Measuring digital development. facts and figures 2019." Technical Report International Telecommunication Union (ITU), 2019.

[2] I. del Portillo, S. Eiskowitz, E. F. Crawley, and B. G. Cameron, "Connecting the other half: Exploring options for the 50\% of the population unconnected to the internet," Telecommunications Policy, vol. 45 , no. 3, pp. 1-34, 2021. 
[3] "[online] available at: https://en.wikipedia.org/wiki/starlink."

[4] F. Fidler, M. Knapek, J. Horwath, and W. R. Leeb, "Optical communications for high-altitude platforms," IEEE Journal of Selected Topics in Quantum Electronics, vol. 16, no. 5, pp. 1058-1070, 2010.

[5] J. Ye, S. Dang, B. Shihada, and M.-S. Alouini, "Space-air-ground integrated networks: Outage performance analysis," IEEE Transactions on Wireless Communications, vol. 19, no. 12, pp. 7897-7912, 2020.

[6] N. Saeed, H. Almorad, H. Dahrouj, T. Y. Al-Naffouri, J. S. Shamma, and M.-S. Alouini, "Point-to-point communication in integrated satelliteaerial $6 \mathrm{~g}$ networks: State-of-the-art and future challenges," IEEE Open Journal of the Communications Society, pp. 1-1, 2021.

[7] S. C. Arum, D. Grace, and P. D. Mitchell, "A review of wireless communication using high-altitude platforms for extended coverage and capacity," Computer Communications, vol. 157, pp. 232-256, 2020.

[8] "Haps mobile inc., [online] available at: https://www.hapsmobile.com."

[9] L. Gupta, R. Jain, and G. Vaszkun, "Survey of important issues in uav communication networks," IEEE Communications Surveys Tutorials, vol. 18, no. 2, pp. 1123-1152, 2016.

[10] A. Fotouhi, H. Qiang, M. Ding, M. Hassan, L. G. Giordano, A. GarciaRodriguez, and J. Yuan, "Survey on uav cellular communications: Practical aspects, standardization advancements, regulation, and security challenges," IEEE Communications Surveys Tutorials, vol. 21, no. 4 pp. 3417-3442, 2019.

[11] A. A. Khuwaja, Y. Chen, N. Zhao, M.-S. Alouini, and P. Dobbins, "A survey of channel modeling for uav communications," IEEE Communications Surveys Tutorials, vol. 20, no. 4, pp. 2804-2821, 2018.

[12] M. Li, Y. Hong, C. Zeng, Y. Song, and X. Zhang, "Investigation on the uav-to-satellite optical communication systems," IEEE Journal on Selected Areas in Communications, vol. 36, no. 9, pp. 2128-2138, 2018.

[13] J. Ma, K. Li, L. Tan, S. Yu, and Y. Cao, "Performance analysis of satellite-to-ground downlink coherent optical communications with spatial diversity over gamma gamma atmospheric turbulence," Appl. Opt., vol. 54, pp. 7575-7585, Sep 2015.

[14] M. Usman, H.-C. Yang, and M.-S. Alouini, "Practical switching-based hybrid fso/rf transmission and its performance analysis," IEEE Photonics Journal, vol. 6, no. 5, pp. 1-13, 2014.

[15] J. Libich, M. Komanec, S. Zvanovec, P. Pesek, W. O. Popoola, and Z. Ghassemlooy, "Experimental verification of an all-optical dual-hop gbit/s free-space optics link under turbulence regimes," Opt. Lett., vol. 40, pp. 391-394, Feb 2015.

[16] E. Zedini, H. Soury, and M.-S. Alouini, "Dual-hop fso transmission systems over gamma-gamma turbulence with pointing errors," IEEE Transactions on Wireless Communications, vol. 16, no. 2, pp. 784-796, 2017.

[17] S. Sharma, A. S. Madhukumar, and R. Swaminathan, "Switching-based cooperative decode-and-forward relaying for hybrid fso/rf networks," IEEE/OSA Journal of Optical Communications and Networking, vol. 11, no. 6, pp. 267-281, 2019

[18] R. Swaminathan, S. Sharma, and A. S. MadhuKumar, "Performance analysis of haps-based relaying for hybrid fso/rf downlink satellite communication," in 2020 IEEE 91st Vehicular Technology Conference (VTC2020-Spring), pp. 1-5, 2020.

[19] S. R., S. Sharma, N. Vishwakarma, and A. Madhukumar, "Hapsbased relaying for integrated space-air-ground networks with hybrid fso/rf communication : A performance analysis," IEEE Transactions on Aerospace and Electronic Systems, pp. 1-1, 2021.

[20] M. Siddharth, S. Shah, and S. R., "Outage analysis of adaptive combining scheme for hybrid fso/rf communication," in 2020 National Conference on Communications (NCC), pp. 1-6, 2020.

[21] S. Shah, M. Siddharth, N. Vishwakarma, R. Swaminathan, and A. S. Madhukumar, "Adaptive-combining-based hybrid fso/rf satellite communication with and without haps," IEEE Access, vol. 9, pp. 81492-81511, 2021.

[22] T. V. Nguyen, H. D. Le, N. T. Dang, and A. T. Pham, "Average transmission rate and outage performance of Relay-Assisted satellite hybrid FSO/RF systems." 2021 International Conference on Advanced Technologies for Communications(ATC) (ATC'21), 2021. [Online]. Available: https://doi.org/10.36227/techrxiv.14971587.v1.

[23] H. D. Le and A. T. Pham, "Level crossing rate and average fade duration of satellite-to-uav fso channels," IEEE Photonics Journal, vol. 13, no. 1, pp. 1-14, 2021.

[24] J. Li, Y. Yao, G. Wu, J. Hou, W. Yu, B. Liu, and J. Liu, "Broadband laser doppler frequency shift emulator for satellite laser communication," IEEE Photonics Journal, vol. 11, no. 6, pp. 1-12, 2019.

[25] F. Yang, J. Cheng, and T. A. Tsiftsis, "Free-space optical communication with nonzero boresight pointing errors," IEEE Transactions on Communications, vol. 62, no. 2, pp. 713-725, 2014.
[26] "Attenuation due to clouds and fog." document Rec. ITU-R P-840-6, 2013.

[27] M. Simon and M.-S. Alouini, Digital Communication over Fading Channels, 2nd Edition. Wiley Publishers, 2005.

[28] P. V. Trinh, T. Cong Thang, and A. T. Pham, "Mixed mmwave rf/fso relaying systems over generalized fading channels with pointing errors," IEEE Photonics Journal, vol. 9, no. 1, pp. 1-14, 2017.

[29] H. Kaushal and G. Kaddoum, "Optical communication in space: Challenges and mitigation techniques," IEEE Communications Surveys Tutorials, vol. 19, no. 1, pp. 57-96, 2017.

[30] E. Erdogan, I. Altunbas, G. K. Kurt, M. Bellemare, G. Lamontagne, and H. Yanikomeroglu, "Site diversity in downlink optical satellite networks through ground station selection," IEEE Access, vol. 9, pp. 3117931190, 2021.

[31] L. D. Hoang, T. Nguyen, and A. Pham, "Cloud attenuation statistical model for satellite-based fso communications," IEEE Antennas and Wireless Propagation Letters, pp. 1-1, 2021.

[32] T. V. Nguyen, H. D. Le, T. V. Pham, and A. T. Pham, "Link availability of satellite-based fso communications in the presence of clouds and turbulence," IEICE Communications Express, vol. 10, 2021.

[33] M. Alzenad, M. Z. Shakir, H. Yanikomeroglu, and M.-S. Alouini, "Fsobased vertical backhaul/fronthaul framework for $5 \mathrm{~g}+$ wireless networks," IEEE Communications Magazine, vol. 56, no. 1, pp. 218-224, 2018.

[34] T. V. Nguyen, T. V. Pham, N. T. Dang, and A. T. Pham, "Uavbased fso systems using sc-qam signaling over fading channels with misalignment," in 2020 IEEE 92nd Vehicular Technology Conference (VTC2020-Fall), pp. 1-5, 2020

[35] M. Q. Vu, T. V. Pham, N. T. Dang, and A. T. Pham, "Outage performance of hap-uav fso links with gaussian beam and uav hovering," in 2020 IEEE 92nd Vehicular Technology Conference (VTC2020-Fall), pp. 1-5, 2020.

[36] A. Prudnikov, Y. Brychkov, and O. Marichev, Integrals, and seriers: volume 3 more special function, vol. 3. Gordon and Breach Science Publishers, 1986.

[37] V. Adamchik and O. Marichev, "the algorithm for calculating integrals of hyperfeometric type functions and its realization in reduce system," Proc. International Conference on Symbolic and Algebraic Computation, pp. 212-224, 1990

[38] V. V. Mai and A. T. Pham, "Cross-layer designs and analysis of adaptiverate transmission and arq for free-space optical communications," IEEE Photonics Journal, vol. 8, no. 1, pp. 1-15, 2016. 